\title{
Original Article \\ Cadaveric Study of Lung Anatomy: A Surgical Overview
}

\author{
Arthi Ganapathy, Rati Tandon, Monica Baxla, Saroj Kaler* \\ Department of Anatomy, All India Institutes of Medical Sciences, New Delhi, India \\ Address for correspondence: Dr. Saroj Kaler, Department of Anatomy, First floor, Teaching Block, All \\ India Institutes of Medical Sciences, New Delhi, India. E-mail: sarojkaler@gmail.com
}

\begin{abstract}
Context: Thorough knowledge of variations in lung anatomy is of prime significance during surgical procedures of lungs. Arrangement of structures in lung hilum acts as a guide in performing such procedures. Normal pattern of arrangement of hilar structures in the right lung is eparterial bronchus, pulmonary artery, hyparterial bronchus, and pulmonary veins from above downward. In the left lung, it is pulmonary artery, principal bronchus, and pulmonary vein from above downward. Arrangement of hilar structures from anterior to posterior in both lungs is pulmonary vein, pulmonary artery, and principal bronchus. Aims: This study aims to report the variations in hilar anatomy (arrangement and number) of lungs. Materials and Methods: A total of 75 adult formalin-fixed cadaveric lungs from the Department of Anatomy, AIIMS, New Delhi, were observed for variations in lobar anatomy. Arrangement of pulmonary hilar structures observed and variations recorded. Results: Among 75 adult lung specimens observed, 36 were the right and rest the left lung. Normal pattern of the arrangement of hilar structures was seen in 22 right and 23 left lungs. Rest of the lung specimens (14 right and 16 left) showed variations in hilar anatomy. Some of them showed alterations in sequence of the arrangement of pulmonary artery, pulmonary veins, bronchus, and others in number of these structures. Seven right lung specimens showed only two lobes with an oblique fissure dividing them and one left lung showed three lobes. Conclusion: Alterations in pattern of the arrangement of structures in lung hilum are quite frequent. A compromise in knowledge of such variations will result in inadvertent intraoperative complications.
\end{abstract}

Publication history: Received: 22-09-2018

Accepted: 10-11-2018

Published: 11-11-2018

Keywords: Fissures, Hilum, Lobes, Pulmonary

Editors: Dr. Ahmad

Farrokhi and Dr. Varshil Mehta

Copyright: Ganapathy A, Tandon R, Baxla M, Kaler $\mathrm{S}$. This is an open access article distributed under the terms of the Creative Commons Attribution License CC-BY 4.0., which permits unrestricted use, distribution, and reproduction in any medium, provided the original author and source are credited.

Funding: NIL Conflict of Interest: NIL

\section{Introduction}

Each lung consists of two lobes on the left and three on the right which lies within its own side of thoracic cavity and is surrounded by visceral layer of the pleura. ${ }^{[1]}$ Each has a principal bronchus, one pulmonary artery and two pulmonary veins. All undergo subdivisions within the lung substance, together with bronchial vessels, nerves, and lymphatics. ${ }^{[1]}$ Hilum of lung is present on its mediastinal surface. Pulmonary root connects medial surface of lung to heart and trachea. It is formed by a group of structures which enter or leave hilum. There is a fixed pattern of the arrangement of these structures at the hilum. In the right lung, the arrangement of hilar structures from above downward includes eparterial bronchus, pulmonary artery, hyparterial bronchus, and superior and inferior pulmonary veins. While on the left side the arrangement of structures at the hilum includes pulmonary artery, principal bronchus and the pulmonary vein from above downwards. At both hila, the arrangement of structures from anterior to posterior is pulmonary vein, pulmonary artery, and principal bronchus. Bronchial vessels are the posterior-most structures at both hila. A thorough knowledge of variations in lung anatomy is of prime significance during surgical procedures such as lobectomy, pneumonectomy, and segmentectomy of lungs. ${ }^{[2]}$ The arrangement of structures at lung hilum acts as a guide in performing such procedures. Though there are few studies published regarding variations in lobes and fissures of the lungs, the literature is yet scarce about the structures at hilum of lungs. ${ }^{[2-4]}$. Hence, the aim of the present 
study was to document variations in hilar anatomy (arrangement and number) of lungs and variations in fissures and lobes of lungs if any.

\section{Materials and Methods}

A total of 75 adult formalin-fixed cadaveric lungs from the Department of Anatomy, AlIMS, New Delhi, were observed for variations in lobar anatomy. Arrangement of pulmonary hilar structures was meticulously observed. Any deviation in normal pattern of presentation was recorded and documented in the form of photographs. Lungs which exhibited pathological changes at hilar region and mutilations were excluded from the study.

\section{Results}

Among the 75 lungs observed, $36(48 \%)$ were the right lungs and 39 (52\%) were the left lungs. Normal pattern of the arrangement of hilar structures was seen in 22 right (61.11\%) and 23 left (58.97\%) lungs. Variation in pattern of the arrangement of hilar structures was observed in 14 right (38.88\%) and 16 left (41.02\%) lungs. Variations were seen in one of the following ways:

- Deviation from the number of structures present in hilum.

- Deviation from normal pattern of the arrangement of structures in hilum.

- Variations in the number of lobes in lung.

Variations were more common in the left lung. All the variations are summarized in Table 1 . Structure which showed the highest variation at both the lung hila was the pulmonary vein. More than two pulmonary veins were seen in $13(33.33 \%)$ and $14(38.88 \%)$ of the right lungs. Multiple bronchi at the hilum were seen in $6(15.38 \%)$ left lungs. Two right lungs (5.55\%) showed three bronchi at the hilum and two (5.55\%) of them showed only one bronchus at the hilum. More than one pulmonary artery was seen in both the lungs but was more common in the right lungs as depicted in Table 1. Deviation from normal pattern of the arrangement of structures was seen in one (2.56\%) left lung and eight (22.22\%) right lungs. The left lung showed the arrangement of structures from anterior to posterior as pulmonary artery, principal bronchus, and pulmonary vein. Six of the right lungs showed the arrangement of structures from above downward as pulmonary artery, bronchus, and pulmonary vein. Two of the right lungs showed the arrangement of structures from above downward as bronchus, pulmonary artery, and pulmonary vein. Variations in lobar anatomy were seen in only $6(8 \%)$ of lungs. They were seen more frequently in the left compared to the right lungs. One right lung specimen $(2.7 \%)$ showed only two lobes with an incomplete oblique fissure dividing them. Three of the left lungs showed three lobes and the other two left lungs showed an incomplete oblique fissure. The variations observed have been displayed in Figure 1.
Table 1: The presentation of various structures in the hilum of the right and left lungs

Variable presentation of structures at lung hila

\begin{tabular}{lc} 
Presence of more than two pulmonary veins & \\
Right lung & $13(33.33 \%)$ \\
Left lung & $14(38.88 \%)$ \\
Multiple lobar bronchi & \\
Right lung (>2) & $2(5.55 \%)$ \\
Left lung (>1) & $6(15.38 \%)$ \\
Single bronchus in the right lung & $2(5.55 \%)$ \\
Multiple pulmonary arteries & $7(19.44 \%)$ \\
Right lung & $3(7.69 \%)$ \\
Left lung & $8(22.22 \%)$ \\
Deviation of normal pattern of the arrangement of structures at the hilum \\
Right lung & $1(2.56 \%)$ \\
Left lung & \\
Variations in lobar anatomy & $1(2.77 \%)$ \\
Right lung & $5(12.82 \%)$ \\
\hline
\end{tabular}

\section{Discussion}

The respiratory system starts developing by day 22 of the intrauterine life when a diverticulum forms as an evagination of the foregut. By day 26-28, the diverticulum immediately bifurcates into two primary bronchial buds. At around $5^{\text {th }}$ week of intrauterine life, the right bronchial bud branches into three secondary bronchial buds and the left bronchial bud branches into two, and by the $6^{\text {th }}$ week, secondary bronchial buds branch into tertiary bronchial buds to form bronchopulmonary segments. ${ }^{[5]}$ In adult, lung pulmonary arteries accompany the airways. Pulmonary veins have a similar number of branches but are separated from airways by alveoli. Pulmonary vessels form in mesenchyme by vasculogenesis. Airways act as a template for the development of blood vessels. ${ }^{[5]}$ Errors in the pattern of pulmonary branching (branching morphogenesis) during embryonic and early fetal periods result in various defects. This ranges from an abnormal number of pulmonary lobes or bronchial segments to the complete absence of a lung. Branching morphogenesis of the respiratory tree is regulated by reciprocal interaction between endoderm and surrounding mesoderm. ${ }^{[5]}$ If mesenchyme in region of bifurcating bronchial bud is replaced by tracheal mesenchyme, then further branching is inhibited. Replacement of tracheal mesenchyme with that of bronchial bud will stimulate ectopic tracheal budding and branching. Branching morphogenesis is under the control of various growth factors such as retinoic acid, transforming growth factor $\beta$, bone morphogenetic protein, and sonic hedgehog protein.

A thorough literature search showed only two recent studies similar to the present one. Hence, the findings of the present study will be compared with these two studies done by Murlimanju et $a l_{.}^{[6]}$ and George et al. ${ }^{[7]}$ In the present study, variations in hilar structures anatomy were seen in $38.88 \%$ of 

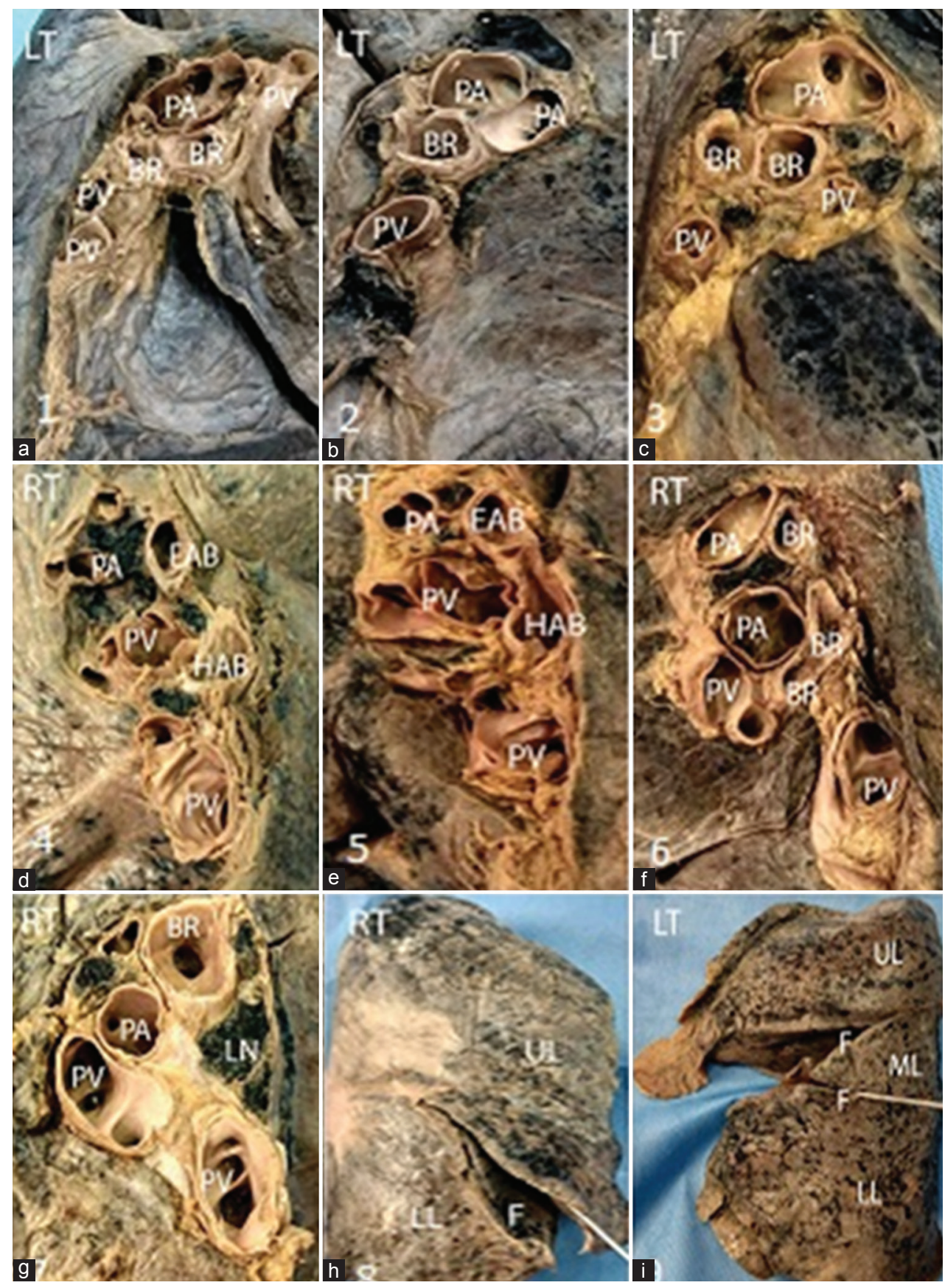

Figure 1: (a) Multiple pulmonary veins in the left lung, (b) Multiple pulmonary arteries in the left lung, (c) Multiple bronchi left lung, (d) Multiple pulmonary veins right lung, (e) Multiple pulmonary arteries right lung, (f) Multiple bronchi right lung, (g) Single bronchus right lung, (h) Incomplete fissure right lung, (i) Three lobes left lung. RT: Right, LT: Left, EAB: Eparterial bronchus, HAB: Hyparterial bronchus, PA: Pulmonary artery, PV: Pulmonary vein, SPV: Superior pulmonary vein, IPV: Inferior pulmonary vein, BA: Bronchial artery, BV: Bronchial vein, LN: Lymph node

the right lungs and $41.02 \%$ of the left lungs. Similar study was done by Murlimanju et al., ${ }^{[6]}$ in 2017, and George et al., in 2014. ${ }^{[7]}$ Murlimanju et al. ${ }^{[6]}$ in their study reported $16.1 \%$ of the right and $48.2 \%$ of the left lungs exhibited variations of structural arrangement. Similar to the previous study in the present variations in pulmonary hilar structures is seen higher in the left lungs. We also found the prevalence of variable hilar structures is higher in the right lungs compared to the previous study.
George et al. ${ }^{[7]}$ reported the presence of two pulmonary arteries in $67.69 \%$ of the right lungs and three pulmonary arteries in $3.07 \%$ of the right lungs. Murlimanju et al. ${ }^{[6]}$ reported the presence of multiple pulmonary arteries in $8.9 \%$ of the right lungs. In the present study, we saw it in $19.44 \%$ of the right lungs which is higher compared to that reported by Murlimanju et al. ${ }^{[6]}$ while much lower compared to George et al. ${ }^{[7]}$ On the left lung, hilum multiple pulmonary arteries were observed in $7.69 \%$ of 
Table 2: Comparison of variations in lobation of the right and left lungs with previous studies

\begin{tabular}{lcccc} 
Study & \multicolumn{3}{c}{ Variations } \\
\cline { 2 - 5 } & $\begin{array}{c}\text { Absence of horizontal } \\
\text { fissure (right lung) }\end{array}$ & $\begin{array}{c}\text { Incomplete oblique } \\
\text { fissure (right lung) }\end{array}$ & $\begin{array}{c}\text { Incomplete oblique } \\
\text { fissure (left lung) }\end{array}$ & $\begin{array}{c}\text { Multiple lobes } \\
\text { (left lung) }\end{array}$ \\
\hline Mamatha et al., 2016 & - & $3(15 \%)$ & $7(35 \%)$ & - \\
George et al., 2014 ${ }^{[7]}$ & $2(23.07)$ & $2(3.07)$ & $11(15.06)$ & $2(2.73)$ \\
Dogan et al., 2015 & $4(1.90)$ & $2(1.42)$ & $2(1.42)$ & $2(1.42)$ \\
Magudam et al., 2014 & $5(12.5 \%)$ & $11(27.5 \%)$ & $5(12.5 \%)$ & - \\
Quadros et al., 2014 & $4(11.11 \%)$ & $2(5.55 \%)$ & $1(2.5 \%)$ & - \\
Present study, 2017 & $1(2.7 \%)$ & $1(2.7 \%)$ & $3(7.69 \%)$ & $2(5.12 \%)$ \\
\hline
\end{tabular}

specimens almost similar to that reported by Murlimanju et al. ${ }^{[6]}$ (5.5\%) and George et al. ${ }^{[7]}(5.47 \%)$. The prevalence of multiple pulmonary veins was seen in $33.33 \%$ of the right lungs which is similar to that reported by George et al. ${ }^{[7]}$ They reported three pulmonary veins in $32.3 \%$ of the right lungs and more than three pulmonary veins in $4.6 \%$ of the right lungs. Murlimanju et al. ${ }^{[6]}$ have reported a very less prevalence of multiple pulmonary veins of $1.8 \%$ in the right lungs. Variations in the prevalence of multiple pulmonary veins (38.88\%) are higher in the left compared to the right lungs. This finding is in concordance with previous studies by Murlimanju et al. ${ }^{[6]}$ (53.6\%) and George et $a l .{ }^{[7]}(80.2 \%)$ showed two pulmonary veins and $19.7 \%$ showed three pulmonary veins. The prevalence of more than two bronchi was seen in $5.5 \%$ of the right lungs and $15.38 \%$ of the left lungs in the present study. Similar to previous studies, the prevalence of multiple bronchi was lower in the right compared to the left lungs. Murlimanju et al. ${ }^{[6]}$ observed it in $3.6 \%$ of the right and $35.2 \%$ of the left lungs, while George et al. ${ }^{[7]}$ reported it in $1.5 \%$ right and $21.9 \%$ of left lungs.

Anomalies of lobation ${ }^{[8]}$ may be produced by fusion of adjacent lobes, occurrence of abnormal fissures, or aplasia or agenesis of a part of lung.

Unlike, the hilar anatomical studies variations in lobation were described by quite a few numbers of authors. The results of the present study are compared with previous in Table 2.

\section{Conclusion}

Variations in arrangement and number of structures in lung hilum are quite frequent. Most frequent variation is the presence of multiple pulmonary veins. The presence of multiple bronchi is more common in the left compared to the right lungs. Multiple pulmonary arteries are more common in the right lungs. Deviation in normal pattern of the arrangement of structures from lung hilum is common in the right lungs. Variations in lobar anatomy of lungs are not quite common. Details of hilar relations to individual lobes and chief variations are important as individual closure of structures at hilum is preferred to mass ligation during pneumonectomy and lobectomy. Identification of anatomical variation during bronchoscopy procedure would prevent complications such as catastrophic bleeding and inadvertent injuries. Lack of knowledge of the variant hilar anatomy may cause misinterpretation of radiological investigations (X-ray and computed tomography). They can be misdiagnosed as hilar masses.

\section{Limitations}

The present study has certain limitations. Hilar structures were not traced through intrapulmonary tissue which could have added to further knowledge on intrapulmonary branching patterns. The other limitation is the relatively smaller sample size. This has prevented us from performing an inferential analysis. Further larger studies need to be done to fully understand the variations in the population.

\section{Ethics}

The study was performed in accordance to the Declaration of Helsinki and was ethically accepted by the Institutional Ethical Committee.

\section{References}

1. Standring S. Gray's Anatomy. $40^{\text {th }}$ ed. UK; Elsevier Publications; 2008. p. 1819-24.

2. Dogan NU, Uysal II, Demirci S, Dogan KH, Kolcu G. Major anatomic variations of pulmonary fissures and lobes on postmortem examination. Acta Clin Croat 2015;54:201-7.

3. Magudam A, Dixit D, Bhimalli S. Fissures and lobes of lung - An anatomical study and its clinical significance. Int J Cur Res Rev 2015;7:8-12.

4. Quadros LS, Palanichamy R, D'souza AS. Variations in the lobes and fissures of lungs - A study in South Indian lung specimens. Eur J Anat 2014;18:16-20.

5. Schoenwolf GC, Bleyl SB, Brauer PR, Francis-West PH. Larsen Human Embryology. $4^{\text {th }}$ ed. New York; Edinburgh: Churchill Livingstone, Elsivier Publications; 2015. P. 324-5.

6. Murlimanju BV, Massand A, Madhyastha S, Pai MM, Prabhu LV, Saralaya VV, et al. Anatomical variations of the arrangement of structures at the pulmonary hilum: A cadaveric study. Surg Radiol Anat 2017;39:51-6.

7. George BM, Nayak SB, Marpalli S. Morphological variations of the lungs: A study conducted on Indian cadavers. Anat Cell Biol 2014;47:253-8.

8. Hollisnshed H. Textbook of Anatomy. $2^{\text {nd }}$ ed. New York: Harper and Row; 1970. p. 507-10.

9. Mamatha Y, Murthy CK, Prakash BS. Study of morphological variations of fissures and lobes of lung. Int J Anat Res 2016;4:1874-7. 